\title{
Clinical outcomes of bending versus non-bending of the plate hook in acromioclavicular joint dislocation
}

\author{
Min Su Joo, Hoi Young Kwon, Jeong Woo Kim \\ Department of Orthopedic Surgery, Wonkwang University Hospital, Iksan, Korea
}

\begin{abstract}
Background: We aimed to assess the effect of plate hook bending in treatment of acromioclavicular (AC) dislocation by analyzing clinical and radiological results according to the angle of the plate hook (APH).

Methods: This was a retrospective, observational, case-control study including 76 patients with acute AC joint dislocation that were divided into two groups according to treatment with bent or unbent plate hook. The visual analog scale (VAS), the American Shoulder and Elbow Surgeons (ASES) shoulder score, and range of motion (ROM) were evaluated as clinical outcomes. Comparative coracoclavicular distance (CCD) was measured to evaluate radiological outcomes.

Results: While the VAS and ASES of the bending group at 4 months after surgery were significantly higher ( $\mathrm{p}=0.021$ and $\mathrm{p}=0.019)$, the VAS and ASES of the bending group at other periods and ROM of the bending group showed no significant difference. The initial CCD decreased from $183.2 \% \pm 25.4 \%$ to $114.3 \% \pm 18.9 \%$ at the final follow-up in the bending group and decreased from $188.2 \% \pm 34.4 \%$ to $119.1 \% \pm 16.7 \%$ in the non-bending group, with no statistical difference $(\mathrm{p}=0.613)$. The changes between the initial and post-metal removal CCD were $60.2 \% \pm 11.2 \%$ and $57.3 \% \pm 10.4 \%$, respectively, with no statistical difference $(p=0.241)$. The non-bending group showed greater subacromial osteolysis (odds ratio, 3.87). Pearson's coefficients for the correlation between APH and VAS at 4 months after surgery and for that between APH and ASES at 4 months after surgery were 0.74 and -0.63 ( $\mathrm{p}=0.027$ and $\mathrm{p}=0.032$ ), respectively.

Conclusions: The APH was associated with improved postoperative pain and clinical outcomes before implant removal and with reduced complications; therefore, plate hook bending is more useful clinically during plate implantation.
\end{abstract}

Keywords: Acromioclavicular joint; Acromioclavicular injury; Plate hook bending

\section{INTRODUCTION}

Acromioclavicular (AC) joint injury accounts for $9 \%-12 \%$ of shoulder injuries that are caused by falling on outstretched hands [1]. Recently, as the frequency of traffic, industrial, and falling accidents has increased, the frequency of AC joint injuries also has increased. AC joint dislocations are classified according to the Rockwood classification [2]. Despite controversies, surgical treatment is considered in injuries more severe than type 3 [3-5]. Among the previously introduced treatment options, such as pinning, tension band wiring, washer screw, and clavicular hook plate, the best option remains controversial [6-8].

After being introduced by Hackenbruch, open reduction and internal fixation (ORIF) with a hook plate gradually grew to be

Received: July 23, $2021 \quad$ Revised: September 1, $2021 \quad$ Accepted: September 5, 2021

Correspondence to: Jeong Woo Kim

Department of Orthopedic Surgery, Wonkwang University Hospital, 344-2 Shinyong-dong, Iksan, Jeollabuk-do, Korea

Tel: +82-63-859-1360, Fax: +82-63-852-9329, E-mail: serina@wonkwang.ac.kr, ORCID: https://orcid.org/0000-0002-0828-7179

Financial support: None.

Conflict of interest: None.

Copyright@ 2021 Korean Shoulder and Elbow Society.

This is an Open Access article distributed under the terms of the Creative Commons Attribution Non-Commercial License (http://creativecommons.org/licenses/by-nc/4.0/) which permits unrestricted non-commercial use, distribution, and reproduction in any medium, provided the original work is properly cited. 
considered the ideal method as it could easily maintain reduction without affecting the injury to the AC joint space, and it allows normal biomechanical rotation between the clavicle and scapula with non-rigid fixation. This allows long-term retention of the plates, which can help in appropriate recovery of the coracoclavicular (CC) ligament [9-12].

As the use of hook plates increased, complications related to internal fixation also increased. Complications such as subacromial impingement syndrome, subacromial erosion, metal failure, need for secondary surgery for metal removal, wide skin incision, widening of the hook hole, and pain due to metal irritation when retained for long-term and stress fractures have been reported $[7,13,14]$. In particular, postoperative pain was reported as a comprehensive sign of various complications that affected the shoulder. According to a report, $14 \%$ of subjects who underwent surgery with hook plates for distal clavicular fracture experienced postoperative pain [15].

Studying the cause of postoperative pain and its prevention methods has become an important task. Many studies have reported that these are related to the position of the hook plate, subacromial impingement syndrome, and functional exercise [11,12].

According to Ko [14], if the tip of the plate hook was pointing upward to the subacromion, such as in Wolter's crook plates, the pain resulting from impingement and metal irritation could not be overlooked, as three of 11 patients in that study complained of persistent pain before metal removal. In this study, we aimed to determine the effect of plate hook bending by analyzing clinical and radiological results based on the shape of the plate hook.

\section{METHODS}

We conducted this study in compliance with the principles of the
Declaration of Helsinki. The study's protocol was reviewed and approved by the Institutional Review Board of Wonkwang University Hospital (IRB No. WKUHIRB 2021-07-032), and agreement of the patient was exempted as it was a retrospective study.

\section{Subjects}

This study was retrospectively performed on patients who had acute (less than 2 weeks) Rockwood type III, IV, or V AC joint dislocation and were treated with hook plate fixation at our hospital from 2011 to 2019. Among 103 patients, three who were younger than 18 years, 17 with concomitant injury, four with abnormal range of motion (ROM) before injury, and three who could not be followed up after metal removal were excluded from the study according to exclusion criteria. A total of 76 patients was included in the study. Most of the injuries were low energy, such as slipping and sports injuries, or high energy, such as falls and traffic accidents. As a result of the retrospective analysis, 31 cases underwent surgery without bending of the plates prior to and in the year 2014. As patients complained of complications such as postoperative pain, we performed downward plate bending in 45 cases from the year 2015 onward.

\section{Surgical Technique}

Surgery was performed under general anesthesia in the supine position. A senior surgeon (JWK) performed all the surgeries. The incision was made from the lateral tip of the acromion to the medial side of the coracoid process. After subcutaneous dissection, the AC joint and CC ligament were exposed. The site for the insertion of the hook plate (Arbeitsgemeinschaft für osteosynthesefragen hook locking compression plate [LCP]; Synthes, Solothurn, Switzerland; LCP clavicle hook plate, Taeyeon Medical, Seoul, Korea) was determined to be on the soft tissue posteri-
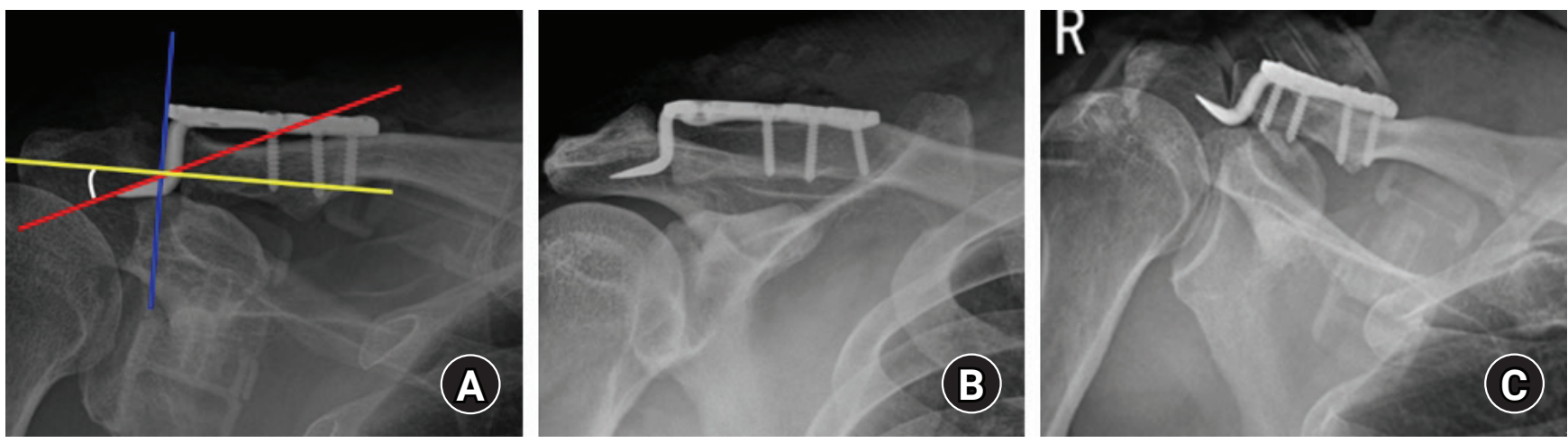

Fig. 1. (A) Plate inclined $15^{\circ}$ downward. Angle of plate hook is the angle between the tip and longitudinal post, determined as the angle (white curved line) between the perpendicular line (yellow line) of the lateral edge of the plate (blue line) and the superior edge of the tip of the hook (red line). (B) Anteroposterior view of the shoulder radiograph with a bent plate hook. (C) Anteroposterior view of the shoulder radiograph with an unbent plate hook. 
or to the AC joint. From 2015, a bending iron and flier were used to bend the plate hook approximately $15^{\circ}$ downward by bending the tip and longitudinal post of the plate hook (Fig. 1). The plate was inserted to reach the lower part of the acromion. Downside pressure on the clavicular part of the plate was used for reduction. Furthermore, the acromion helped the reduction, as it worked as a lever. Before inserting the screws, the authors used a radiographic image intensifier to confirm the adequacy of reduction by evaluating the positions of the acromion, clavicle, and the hook fixed on the acromion.

The hook plates were removed 4 months after ORIF [16,17]. The patients performed passive forward elevation and pendulum exercises using Kenny-Howard braces from postoperative day 1 (POD 1) and then active exercise with the braces from POD 3 weeks. They were allowed to return to their everyday lives without strenuous exercise and without the braces starting at POD 6 weeks.

\section{Hook Plate Factors}

We performed a retrospective analysis by dividing the subjects into bending and non-bending groups. The angle of the plate hook (APH), that of the tip and longitudinal post of the hook plate, determined as the angle between the perpendicular line of the lateral edge of the plate and the superior edge of the tip of the hook, was evaluated in the two groups.

\section{Clinical Evaluation}

The visual analog scale (VAS) was evaluated before surgery, 4 months after the surgery (just before metal removal), 6 months after metal removal, and at the final follow-up (1 year after metal removal).

\section{Radiological Evaluation}

To evaluate AC joint reduction after the surgery, the CC distance (the height between the upper border of the coracoid process and the inferior cortex of the clavicle) was measured before ORIF and at 6 months to 1 year after metal removal. The percentage increase of the affected side compared to that of the unaffected side was calculated (comparative coracoclavicular distance [CCD]). Subluxation, re-dislocation, subacromial osteolysis, metal displacement, or peri-implant fractures were evaluated as postoperative radiological complications. Subluxation was defined as the clavicle being less than $50 \%$ displaced compared to the height of the acromion; displacement greater than $50 \%$ was defined as dislocation [18].

\section{Statistical Analysis}

The paired t-test was used to assess differences between the preoperative and postoperative outcomes in each group, while an independent t-test was used to compare results between the groups. The chi-square test and Fisher's exact test were used to assess categorical data of the injury type. Pearson's coefficient was used to describe the correlations of APH and other outcomes with statistically significant difference between the two groups. The SPSS ver. 12.0 (SPSS Inc., Chicago, IL, USA) was used for all statistical analyses. The significance level for all analyses was set at $\mathrm{p}<0.05$.

\section{RESULTS}

The mean age of the subjects was 52.9 years (range, $24-82$ years) for the bending group and 56.4 years (range, 29-81 years) for the non-bending group. The American Shoulder and Elbow Surgeons (ASES) shoulder score and ROM were used to evaluate

Table 1. Demographics of the subgroups

\begin{tabular}{|c|c|c|c|}
\hline Variable & Bending group & Non-bending group & p-value \\
\hline Case & 45 & 31 & - \\
\hline Type of hook plate (AO/Taeyeon) & $14: 31$ & $13: 18$ & 0.333 \\
\hline Sex (male:female) & $26: 19$ & $17: 14$ & 0.799 \\
\hline Age (yr) & $52.9(24-82)$ & $56.4(29-81)$ & 0.254 \\
\hline Angle of plate hook $\left(^{\circ}\right)$ & $2.8 \pm 1.2$ & $21.2 \pm 6.9$ & 0.031 \\
\hline Follow-up period after metal removal (mo) & $16.1 \pm 1.4$ & $15.7 \pm 1.1$ & 0.352 \\
\hline Injury type & & & 0.862 \\
\hline High-energy (fall down, traffic accident) & 37 & 25 & \\
\hline Low-energy (slip down) & 8 & 6 & \\
\hline Classification & & & 0.976 \\
\hline Rockwood III & 14 & 9 & \\
\hline Rockwood IV & 6 & 4 & \\
\hline Rockwood V & 25 & 18 & \\
\hline
\end{tabular}

Values are presented as number, median (range), or mean \pm standard deviation. A p-value $<0.05$ was considered significant. 
functional outcomes at every regular follow-up. Metal removal was performed $4.1 \pm 0.4$ months after surgery. The mean follow-up periods were $16.1 \pm 1.4$ and $15.7 \pm 1.1$ months for the bending and non-bending groups, respectively. The demographic data are shown in Table 1. The number of low-energy injuries was 14, while that of high-energy injuries was 62. The mean APH of the bending group was $21.2^{\circ} \pm 6.9^{\circ}$, while that of the non-bending group was $2.8^{\circ} \pm 1.2^{\circ}(\mathrm{p}=0.031)$.

In the clinical outcomes, the VAS of the bending group improved from $3.8 \pm 0.9$ before surgery to $2.2 \pm 0.4,1.2 \pm 0.2$, and $1.1 \pm 0.1$ at 4 months after surgery (just before metal removal), 6 months after metal removal, and at the final follow-up (1 year after metal removal), respectively; while that for the non-bending group improved from $3.7 \pm 0.6$ to $3.5 \pm 0.3,1.4 \pm 0.3$, and $1.2 \pm 0.2$, respectively. In particular, the VAS at 4 months after surgery was better in the bending group than in the non-bending group with statistical significance $(\mathrm{p}=0.021)$ but did not show a statistically significant difference at 6 months after metal removal or at the final follow-up (Table 2).

The ASES score in the bending group improved from $66.2 \pm 49.1$ at 4 months after the surgery to $83.2 \pm 44.8$ at 6 months after metal removal and $85.1 \pm 42.3$ at the final follow-up; in the non-bending group, it improved from $56.2 \pm 39.8$ at 4 months after the surgery to $79.2 \pm 9.8$ and $82.1 \pm 30.1$. The ASES at 4 months after surgery was better in the bending group than in the non-bending group with statistical significance $(\mathrm{p}=0.019)$ but did not show a statistically significant difference at 6 months after metal removal or at the final follow-up (Table 2).

In ROM, active forward elevation angles at 4 months after surgery were $121.1^{\circ} \pm 13.8^{\circ}$ and $105.1^{\circ} \pm 16.4^{\circ}$ in the bending and non-bending groups, respectively. At 6 months after metal removal, they improved to $141.1^{\circ} \pm 16.4^{\circ}$ and $139.3^{\circ} \pm 15.5^{\circ}$, respectively. At the final follow-up, they were $151.1^{\circ} \pm 12.3^{\circ}$ and $148.7^{\circ} \pm 10.1^{\circ}$, respectively. There was no statistically significant difference at any time point. External rotation, internal rotation, and abduction showed the same tendencies (Table 2).

CCD measurement for evaluation of radiological outcomes was $183.2 \% \pm 25.4 \%$ preoperatively and $114.3 \% \pm 18.9 \%$ at 4 months after surgery in the bending group and $188.2 \% \pm 34.4 \%$ and $119.1 \% \pm 16.7 \%$, respectively, in the non-bending group. The changes in CCD between the initial measurement and the post-metal removal measurement were $60.2 \% \pm 11.2 \%$ in the bending group and $57.3 \% \pm 10.4 \%$ in the non-bending group, with no statistical significance $(\mathrm{p}=0.241)$. The two groups showed decreased post-

Table 2. Comparison of clinical outcomes between the subgroups

\begin{tabular}{|c|c|c|c|}
\hline Variable & Bending group & Non-bending group & $\mathrm{p}$-value \\
\hline \multicolumn{4}{|l|}{ Initial outcome } \\
\hline VAS score & $3.8 \pm 0.9$ & $3.7 \pm 0.6$ & 0.361 \\
\hline \multicolumn{4}{|c|}{ POD 4-month outcome } \\
\hline VAS score & $2.2 \pm 0.4$ & $3.5 \pm 0.3$ & 0.021 \\
\hline ASES score & $66.2 \pm 49.1$ & $56.2 \pm 39.8$ & 0.019 \\
\hline \multicolumn{4}{|c|}{ Post-metal removal outcome } \\
\hline VAS score & $1.2 \pm 0.2$ & $1.4 \pm 0.3$ & 0.235 \\
\hline ASES score & $83.2 \pm 44.8$ & $79.2 \pm 9.8$ & 0.247 \\
\hline \multicolumn{4}{|c|}{ Last FU outcome } \\
\hline VAS score & $1.1 \pm 0.1$ & $1.2 \pm 0.2$ & 0.317 \\
\hline ASES score & $85.1 \pm 42.3$ & $82.1 \pm 30.1$ & 0.251 \\
\hline \multicolumn{4}{|c|}{ Range of motion (POD $4 \mathrm{mo},{ }^{\circ}$ ) } \\
\hline Active FE & $121.1 \pm 13.8$ & $101.5 \pm 14.7$ & 0.341 \\
\hline ER & $55.2 \pm 8.3$ & $54.3 \pm 11.3$ & 0.237 \\
\hline IR & $24.5 \pm 6.9$ & $25.1 \pm 3.8$ & 0.155 \\
\hline Abduction & $75.1 \pm 4.9$ & $64.0 \pm 8.5$ & 0.148 \\
\hline \multicolumn{4}{|c|}{ Range of motion (last FU, ${ }^{\circ}$ ) } \\
\hline Active FE & $151.1 \pm 12.3$ & $148.7 \pm 10.1$ & 0.982 \\
\hline ER & $74.2 \pm 12.3$ & $73.8 \pm 11.7$ & 0.754 \\
\hline IR & $33.2 \pm 5.8$ & $32.7 \pm 6.3$ & 0.894 \\
\hline Abduction & $85.2 \pm 10.4$ & $84.9 \pm 7.4$ & 1.014 \\
\hline
\end{tabular}

Values are presented as mean \pm standard deviation. A p-value $<0.05$ was considered significant.

VAS: visual analog scale, POD: postoperative day, ASES: American Shoulder and Elbow Surgeons, FU: follow-up, FE: forward elevation, ER: external rotation, IR: internal rotation. 
Table 3. Comparison of CCD measurement and complications between the subgroups

\begin{tabular}{lccc}
\hline Variable & Bending group $(\mathrm{n}=45)$ & Non-bending group $(\mathrm{n}=31)$ & $\mathrm{p}$-value \\
\hline Initial CCD (affected:unaffected, \%) & $183.2 \pm 25.4$ & $188.2 \pm 34.4$ & 0.214 \\
Postoperative CCD (\%) & $97.3 \pm 16.2$ & $97.8 \pm 12.7$ & 0.857 \\
Post-metal removal CCD (\%) & $114.3 \pm 18.9$ & $119.1 \pm 16.7$ & 0.613 \\
Change between initial and post-metal removal CCD (\%) & $60.2 \pm 11.2$ & $57.3 \pm 10.4$ & 0.241 \\
Pathology of AC joint (intact:subluxation:re-dislocation) & $35: 9: 1$ & $25: 6: 0$ & 0.701 \\
Subacromial osteolysis & 3 & 8 & 0.020 \\
\hline
\end{tabular}

Values are presented as mean \pm standard deviation or number. A p-value $<0.05$ was considered significant.

CCD: comparative coracoclavicular distance, AC: acromioclavicular.

operative CCD compared to the preoperative states and showed no significant difference between the two groups (Table 3).

After metal removal, nine cases in the bending group and six in the non-bending group showed subluxation, and there was an increasing tendency according to the increase in severity of the injury subtype. Asymptomatic patients underwent conservative treatment. One patient showed symptoms of pain and discomfort and underwent the Weaver-Dunn procedure. One case in the bending group showed re-dislocation, which required refixation with another hook plate (Table 3).

A total of 11 cases showed subacromial osteolysis during the final radiological follow-up with X-rays, with most of these cases $(n=8)$ being in the non-bending group. The odds ratio between the two groups was $3.87(8 / 31: 3 / 45)$, showing significantly increased subacromial osteolysis in the non-bending group $(\mathrm{p}=0.020)$ (Table 3).

Among the outcomes, VAS and ASES at 4 months after surgery and the proportion of the number of patients with subacromial osteolysis showed statistically significant difference between the two groups. The amount of subacromial osteolysis was not quantifiable, thus the odds ratio between the groups were not evaluated. Therefore, we used Pearson's coefficient for the correlation between APH and VAS at 4 months after surgery, along with the correlation between APH and ASES at 4 months after surgery. Pearson's coefficient of the former was $0.74(p=0.027)$, while that of the latter was $-0.63(\mathrm{p}=0.032)$ (Table 4$)$.

Other complications, such as deep infection or nerve injury, were not observed in this study. According to the results of this study, the mean APH of the two groups was different $\left(2.8^{\circ} \pm 1.2^{\circ}\right.$ in the bending group and $21.2^{\circ} \pm 6.9^{\circ}$ in the non-bending group), and this difference in $\mathrm{APH}$ was a factor of significant differences in VAS and ASES at 4 months after surgery (Table 2).

\section{DISCUSSION}

In this study, while APH according to bending of the plate hook
Table 4. Correlations of APH with other outcomes

\begin{tabular}{lcc}
\hline Variable & $\begin{array}{c}\text { Pearson's } \\
\text { coefficient }\end{array}$ & p-value \\
\hline APH and VAS score at 4 months after surgery & 0.74 & 0.027 \\
APH and ASES score at 4 months after surgery & -0.63 & 0.032 \\
\hline
\end{tabular}

A p-value $<0.05$ was considered significant.

APH: angle of plate hook, VAS: visual analog scale, ASES: American Shoulder and Elbow Surgeons.

did not affect postoperative CCD, it was an associated with postoperative pain and clinical outcomes before implant removal and reduced complications such as subacromial osteolysis and impingement.

Postoperative shoulder pain is the most common complication associated with hook plate fixation, which adversely affects postoperative rehabilitation. This suggests the need to study the cause of pain and its prevention. The main cause was suggested to be related to the plate itself, such as impingement between the clavicle and hook, length of the hook tail, and position. Therefore, early metal removal is recommended for fast rehabilitation $[1,19]$.

According to ElMaraghy et al. [20], the hook can injure the subacromial bursa, narrowing the space and decreasing ROM. In this study, patients showed restricted ROM at 4 months after surgery. At the time of the last follow-up after metal removal, compared to at 4 months after surgery, most patients recovered to normal ROM. In contrast, the non-bending group showed significantly inferior results compared to the bending group in VAS and clinical scores at 4 months after ORIF, which was before metal removal. This is suspected to be due to the sharp tip of the hook plate, which irritates the subacromial space. Without bending, the tip of the hook has a high possibility of being upturned, which can increase the stress on the tip of the hook in the subacromial space due to point contact rather than surface contact, leading to aggravation of the bony erosion and shoulder pain. According to a report by $\mathrm{Xu}$ et al. [21], as a mechanical factor, 
APH independently affected the outcome, showing better outcomes in a group with a higher angle. The bending and non-bending groups had differences in APH, which led to significant differences in pain and clinical scores at 4 months after ORIF, with the bending group showing better clinical outcomes.

When hook plates are used without modification, the risk of complications such as subacromial osteolysis, which can lead to pain, increases due to the concentration of stress on both tips of the plate $[21,22]$. In this study, CCD according to hook plate bending did not show a statistically significant difference between the two groups (postop CCD, $\mathrm{p}=0.857$ ) (Table 3), indicating that bending the plate had no effect on postoperative CCD (post-metal removal CCD, p=0.613) (Table 3). Even though there was no significant difference between the groups according to bending itself, the non-bending group showed more severe subacromial osteolysis (8/31) compared to the bending group (3/45) (odds ratio, 3.87).

ORIF with a hook plate can maintain shoulder function based on leverage [16] but can cause complications such as postoperative pain. In particular, decreased APH, caused by not bending the plate, acted as a factor of pain at 4 months after ORIF. However, this can be managed by metal removal, as evidenced by the lack of significant difference in VAS, which was better in the bending group at 4 months after surgery, between the two groups at the last follow-up.

This study has certain limitations as it was performed retrospectively in a single center with a small number of subjects. In addition, two types of implants were used, but analysis between the implants could not be performed due to the small number of samples. Also, the native acromial slope was not considered as a factor. This study, however, is meaningful for evaluating the factors that affect clinical outcomes and complications before and after metal removal.

The APH was associated with improved postoperative pain and clinical outcomes before implant removal and with reduced complications; therefore, plate hook bending is considered useful clinically during plate implantation.

\section{ORCID}

Min Su Joo

Hoi Young Kwon

Jeong Woo Kim

\section{REFERENCES}

1. Di Francesco A, Zoccali C, Colafarina O, Pizzoferrato R, Flami- ni S. The use of hook plate in type III and V acromio-clavicular Rockwood dislocations: clinical and radiological midterm results and MRI evaluation in 42 patients. Injury 2012;43:147-52.

2. Rockwood CA, Matsen FA, Wirth MA, Lippitt SB, Fehringer EV, Sperling JW. Rockwood and Matsen's the shoulder. Philadelphia, PA: Elsevier; 2004. p. 532-7.

3. Dumonski M, Mazzocca AD, Rios C, Romeo AA, Arciero RA. Evaluation and management of acromioclavicular joint injuries. Am J Orthop (Belle Mead NJ) 2004;33:526-32.

4. Kennedy JC, Cameron H. Complete dislocation of the acromio-clavicular joint. J Bone Joint Surg Br 1954;36:202-8.

5. Larsen E, Hede A. Treatment of acute acromioclavicular dislocation: three different methods of treatment prospectively studied. Acta Orthop Belg 1987;53:480-4.

6. Jiang D, Lü S, Hong Y, Cao Y. Effectiveness comparison of triple endobuttons and clavicular hook plate for treatment of fresh acromioclavicular joint dislocation. Zhongguo Xiu Fu Chong Jian Wai Ke Za Zhi 2012;26:1025-8.

7. Kienast B, Thietje R, Queitsch C, Gille J, Schulz AP, Meiners J. Mid-term results after operative treatment of rockwood grade III-V acromioclavicular joint dislocations with an AC-hookplate. Eur J Med Res 2011;16:52-6.

8. Tan H, Wang S, Zhao J, Qian C, Zhou Q, Shi Y. Treatment of fresh tossy type III acromioclavicular joint dislocations and Neer type II distal clavicle fractures with clavicular hook plate. Zhongguo Xiu Fu Chong Jian Wai Ke Za Zhi 2010;24:69-73.

9. Kang KS, Lee HJ, Lee JS, Kim JY, Park YB. Long term follow up results of the operative treatment of the acromioclavicular joint dislocation with a Wolter plate. J Korean Fracture Soc 2009;22: 259-63.

10. Lee KW, Choi YJ, Ahn HS, et al. Treatment of the acromioclavicular joint dislocation using a AO hook plate. J Korean Shoulder Elbow Soc 2009;12:167-72.

11. Mizue F, Shirai Y, Ito H. Surgical treatment of comminuted fractures of the distal clavicle using Wolter clavicular plates. J Nippon Med Sch 2000;67:32-4.

12. Sim E, Schwarz N, Höcker K, Berzlanovich A. Repair of complete acromioclavicular separations using the acromioclavicular-hook plate. Clin Orthop Relat Res 1995;(314):134-42.

13. Habernek H, Weinstabl R, Schmid L, Fialka C. A crook plate for treatment of acromioclavicular joint separation: indication, technique, and results after one year. J Trauma 1993;35:893-901.

14. Ko SH. Minimal incision Wolter plate fixation on the displaced lateral end fracture of the clavicle and the acromioclavicular dislocation. J Korean Shoulder Elbow Soc 2002;5:23-8.

15. Flinkkilä T, Ristiniemi J, Lakovaara M, Hyvönen P, Leppilahti J. Hook-plate fixation of unstable lateral clavicle fractures: a re- 
port on 63 patients. Acta Orthop 2006;77:644-9.

16. Kumar N, Sharma V. Hook plate fixation for acute acromioclavicular dislocations without coracoclavicular ligament reconstruction: a functional outcome study in military personnel. Strategies Trauma Limb Reconstr 2015;10:79-85.

17. An SH, Kim HC, Kim KY, Lee JH, Yoon SH. Complications of hook-plate fixation for distal clavicle fractures. J Korean Fract Soc 2012;25:38-45.

18. Natera-Cisneros L, Sarasquete-Reiriz J, Escolà-Benet A, Rodriguez-Miralles J. Acute high-grade acromioclavicular joint injuries treatment: Arthroscopic non-rigid coracoclavicular fixation provides better quality of life outcomes than hook plate ORIF. Orthop Traumatol Surg Res 2016;102:31-9.
19. Xu D, Luo P, Chen J, et al. Outcomes of surgery for acromioclavicular joint dislocation using different angled hook plates: a prospective study. Int Orthop 2017;41:2605-11.

20. ElMaraghy AW, Devereaux MW, Ravichandiran K, Agur AM. Subacromial morphometric assessment of the clavicle hook plate. Injury 2010;41:613-9.

21. Xu D, Lou W, Luo P, Shi Y, Guo W, Chen J. Risk factors for postoperative shoulder pain of acromioclavicular joint dislocation treat with hook plate. Orthopedics 2020;23:14-7.

22. Wang CZ, Li DL, Mu SX. Case-control study on clavicular hook plate combined with acromiocoracoid ligament transfer in the treatment of acromioclavicular joint dislocation of type Tossy III in young patients. Zhongguo Gu Shang 2012;25:576-9. 